\title{
A Spectrally Efficient PMR System Utilizing Broadcast Service
}

\author{
Ersin Sengül, Student Member, IEEE, Hayrettin Köymen, Senior Member, IEEE, and Yusuf Ziya İder, Member, IEEE
}

\begin{abstract}
Different trunked Private Mobile Radio (PMR) systems have been designed over the last several decades, all of which have symmetric downlink and uplink channel capacities. Due to this symmetry, these systems may not be spectrally efficient in case of different types of services, which are specific to PMR systems, such as group and broadcast calls. In this study, a new asymmetric trunked PMR system comprising a broadband, wide-area downlink and a narrowband cellular uplink, is proposed to achieve a higher spectral efficiency than current digital trunked PMR systems. This system is spectrally more efficient because in group and broadcast calls only a single downlink channel has to be allocated in the downlink part. However, as the number of clusters in the system increases, this advantage relative to PMR systems is lost, since the latter can employ frequency reuse. Spectral efficiency of the proposed asymmetric system (a-PMR) system and a standard TETRA system are compared using numerical case studies against different traffic loads and number of clusters. The optimum point, with respect to number of clusters, up to which the proposed a-PMR system is more efficient, is determined. It is shown that a very large PMR user population can be efficiently served using the proposed a-PMR system. The issues related to implementing such a system are discussed.
\end{abstract}

Index Terms-Digital audio broadcasting, land mobile radio cellular systems, private mobile radio, spectrum efficiency, terrestrial trunked radio.

\section{INTRODUCTION}

$\mathbf{P}$ ROFESSIONAL or Private Mobile Radio (PMR) systems are operated by a wide range of users, such as Police and Fire Services, the utility (Gas, Water and Electricity) and transportation companies, delivery services, and for on-site applications such as in factories and airports [1]. PMR systems must meet certain requirements, which stem from the specific needs of PMR users. Some of these requirements and specific properties of PMR communications are:

1. Traffic generated per user in PMR is relatively low compared to other systems;

2. Call set-up must be very fast in PMR, less than a second;

3. Half-duplex operation can be employed and is preferred in PMR;

4. Different types of calls like broadcast calls and group calls as well as mobile to mobile direct calls are required in PMR;

Manuscript received May 10, 2004; revised January 6, 2005.

E. Sengül is with the Center for Pervasive Communications and Computing, Department of Electrical Engineering and Computer Science, University of California, Irvine, CA 92697 USA (e-mail: esengul@uci.du).

H. Köymen and Y. Z. İder are with the Department of Electrical and Electronics Engineering, Bilkent University, Ankara, Turkey (e-mail: koymen@ ee.bilkent.edu.tr; ider@ee.bilkent.edu.tr.

Digital Object Identifier 10.1109/TBC.2005.852798
5. Preferred mode of trunking is transmission trunking in PMR;

PMR systems have also benefited from the technologies developed for second generation $(2 \mathrm{G})$ cellular systems, and new generation of trunked and digital PMR systems are now being deployed. Alongside the most popular European TETRA and American APCO 25 standards, MPT1327, IDRA, DIMRS, TETRAPOL, EDACS, and GEOTEK-FHMA can be mentioned among the new PMR systems [2]. As well as providing new functions, these modern PMR systems also have improved spectral efficiency, which is a fundamental requirement. These systems have been compared with respect to traffic performance, network access and spectrum efficiency [3], [4]. European Telecommunications Standards Institute (ETSI) has established ten scenarios, which are representative of possible PMR applications, to be used by its workgroups during the comparison of TETRA with other systems [5]. These scenarios cover a wide range of applications, ranging from large metropolitan networks for security services to networks for motorways and to small networks for airport security and ground services.

Convergence of telecommunication and broadcast networks is an important area of investigation and commercial exploitation. Design and implementation of cooperation systems between different services, such as broadcast and mobile communications, whereby the strengths of existing and upcoming systems are combined, comprise the core of these studies [6]. For example, a new standard, which is originally designed for Terrestrial-Digital Video Broadcasting (DVB-T), has recently emerged to support a return path to implement interactive TV services and web sessions, in particular [7]. High bit rate multimedia applications for mobile users have also attracted attention recently [6], [8]. Such systems are asymmetric, which have higher data rates at the downlink. Proposals have been made in which personal access communications systems, GSM and UMTS, are complemented with Terrestrial-Digital Audio Broadcasting (T-DAB) [9], [10] and DVB-T [11]. In these studies broadband downlink is used only for multimedia applications, and voice calls are carried by the narrowband channels. However it may also be possible to achieve spectrum efficiency for the voice communications if a broadband downlink is available.

It appears that incorporation of a broadband wide area downlink for a PMR system may provide significant spectrum efficiency. In particular, the fact that broadcast and group calls constitute a significant portion of the total number of calls is an important factor in this respect. In ETSI scenarios, the types of calls which fall into this category are group calls and open channels calls. The percentage of group calls range 


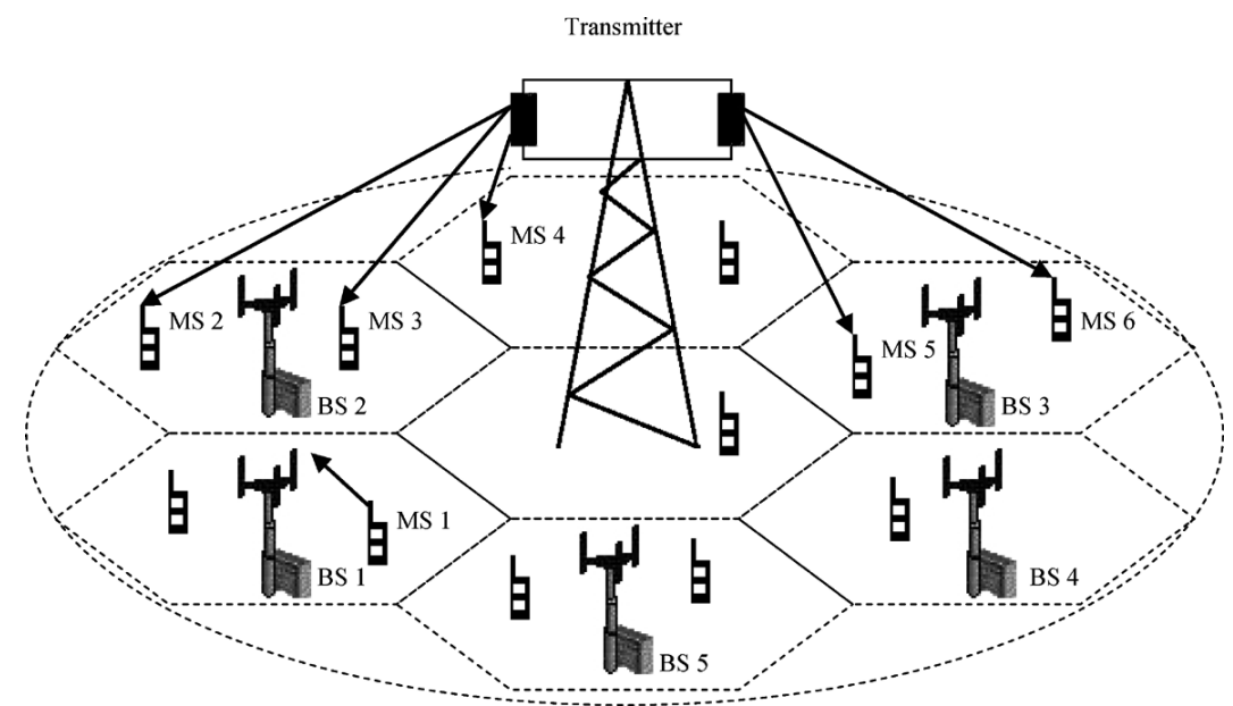

Fig. 1. The generic system architecture of the a-PMR system. Hexagonal cells are assumed to be the coverage areas of the base stations (BS) and the outer circle stands for the coverage area of the downlink transmitter. A typical group call is shown, where mobile station-1 (MS) makes a group call with its group members, MS-2, 3, 4, 5, and 6. MS-1 sends its voice data via the nearest base station BS-1 and all data are broadcast to the other group members via the downlink transmitter, which serves the whole service area.

from $20 \%$ to $50 \%$ of the total number of calls [5]. In five of these scenarios, particularly the ones related to the security and emergency services, there are additional $10 \%$ to $20 \%$ open channel call requirements. When group calls and open channel calls are considered together, they sum up to $60 \%$ in these scenarios.

In this paper, we studied the advantages of employing a broadband wide area downlink in PMR systems as far as spectrum efficiency is concerned. Efficient use of spectrum has many aspects ranging from coexistence of different services to minimization of investment and operation costs. We considered spectrum efficiency from the point of view of data rate capacity per unit bandwidth, under PMR voice communications requirements. Spectrum efficiency is highly dependent on the proportion of different types of calls which have different channel allocation schemes. When a point-to-point individual call is initiated in a digital trunked PMR system, channel allocations are very similar to those in personal mobile communication systems. When a broadcast call is initiated however, a downlink channel must be allocated in all cells in that PMR network. Similarly, when a group call is initiated, a downlink channel is allocated in all cells in which there exists a receiving group member. In this study, we propose an asymmetric PMR (a-PMR) system in which the broadband downlink covers the whole service area. Such a system can provide significant increase in spectral efficiency, because in this case, for a broadcast or group call, only a single downlink channel needs to be allocated.

The standard method for increasing spectrum efficiency in a cellular PMR system is frequency reuse [12]. The a-PMR architecture benefits from trunking efficiency [10] in the downlink and frequency reuse can only be employed in the uplink part. This architecture may not be efficient if the number of cells in the system is increased. In this study we are aiming at determining the optimum point with respect to the number of cells up to which the proposed system is more efficient than current digital PMR systems.

\section{DEFINITION OF A GENERIC a-PMR SYSTEM}

The generic a-PMR system under consideration is composed of a broadband, wide-area downlink and a narrowband cellular uplink. The downlink transmitter or a set of transmitters, as in a Single Frequency Network (SFN) in T-DAB, serves the whole service area. On the other hand, a cellular network, as in the case of a modern PMR network like TETRA or APCO 25, handles the uplink part of any call request.

In the a-PMR system, when a call is initiated in a certain cell, whether a group call or an individual call, one uplink channel is allocated in the initiator cell and one downlink traffic channel is allocated in downlink part. The system architecture is depicted in Fig. 1. The base stations (BS) handle all uplink calls that belong to the corresponding cell, and all downlink transmission in the service area are supported by the broadband and wide-area coverage transmitter. Fig. 2 shows the main conceptual blocks that are needed to implement the downlink part of the proposed a-PMR system. All voice data packets coming from BS's should be first encoded for identification purposes and then encrypted if required. Finally all packets are channel encoded and multiplexed before transmission.

In order to examine the performance of the a-PMR system we have to assign constraints and parameters which are commensurate with existing modern systems. Conventional PMR systems operate in frequency channels of $12.5 \mathrm{kHz}$ or $25 \mathrm{kHz}$ bandwidth in Europe. Modern digital trunked systems adopted compatible frequency channel widths because of inter-operability and compatibility purposes. For example four TDMA communication channels are placed in a $25 \mathrm{kHz}$ frequency channel in TETRA system. On the other hand, APCO $25 \mathrm{em}-$ ploys an FDMA scheme where each communication channel occupies $12.5 \mathrm{kHz}$ bandwidth. In these systems a communication channel can either be used for voice and data traffic or as a control channel. We assumed that the uplink part of the a-PMR has $25 \mathrm{kHz}$ wide frequency channels, each with four communication channels, as in TETRA which is accepted to be the most efficient PMR system in terms of spectral usage 


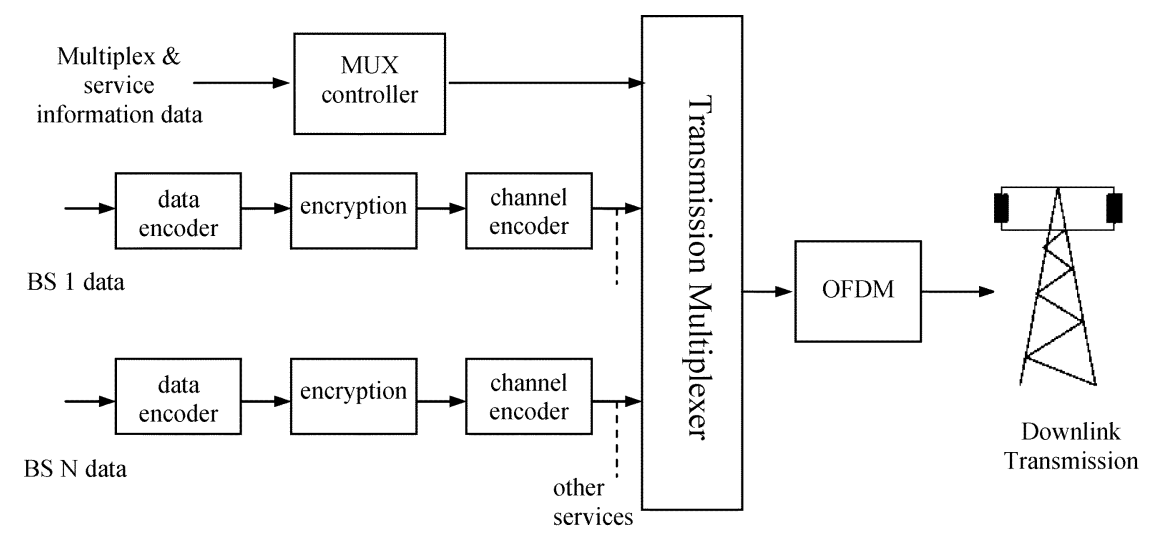

Fig. 2. Conceptual block diagram of the downlink part of the system. The voice data packets coming from all base stations in the service area are encrypted, encoded, and multiplexed to be transmitted by the OFDM based broadband and wide area downlink transmitter.

[13]. We assumed that the uplink part of the a-PMR system has specifications similar to that of TETRA.

Broadband and wide-area coverage downlink systems are mainly used in broadcast applications. One of the new generation terrestrial broadcast systems is T-DAB. T-DAB uses OFDM which is a spectrally efficient multi-carrier digital modulation scheme, offering reliable reception under hostile reception conditions such as multipath propagation [14], [15]. In addition, OFDM allows for intentional multipath, where all the transmitters within a Single Frequency Network operate at the same frequency, thereby achieving wide area coverage. These multipath signals are constructive within the guard interval, strengthening the signal level [14], [15]. T-DAB operates in $1.5 \mathrm{MHz}$ wide frequency channels, which correspond to a gross data rate of $2.3 \mathrm{Mbit} / \mathrm{s}$ and a net rate of typically $1.15 \mathrm{Mbits} / \mathrm{s}$ [16]. It is shown in this paper that the traffic load of a large and crowded PMR network can easily be accommodated in the capacity offered by T-DAB. In this study, for the purpose of numerical simulations and quantitative comparisons we adopted the parameters and capacities similar to those of T-DAB for the downlink part.

The data rate required for voice transmission depends on the compression algorithm used and overheads necessary for error correction and channel coding scheme employed. For example a total of $7.2 \mathrm{kbit} / \mathrm{s}(4.567 \mathrm{kbit} / \mathrm{s}$ for voice and 2.633 for channel coding) is used in TETRA, while $9.6 \mathrm{kbits} / \mathrm{s}$ is common for CELP voice coding with $1 / 2$ rate error control coding [17]. To find a conservative equivalent voice channel capacity for the broadband downlink part, we considered the relatively larger measure of $9.6 \mathrm{kbit} / \mathrm{s}$. With this gross data rate for voice transmission, the total capacity offered by $1.5 \mathrm{MHz}$ channel of T-DAB is able to handle 240 simultaneous voice calls. In this paper we studied the spectrum efficiency of a-PMR for different amounts of total downlink channel capacity up to 240 traffic channels in the service area instead of 252 channels as predicted by net data rates.

\section{Comparative Results FOR the SPECTRUM REQUIREMENTS OF THE a-PMR AND TETRA SYSTEMS}

\section{A. Basic Definitions and Assumptions}

In order to demonstrate the spectrum efficiency achieved by the a-PMR system, we compared the proposed system with TETRA. In particular, we determined the optimum point, with respect to number of cells, up to which the proposed a-PMR system is spectrally more efficient than TETRA system. In order to compare the spectral efficiencies of a-PMR and TETRA, a metropolitan area is assumed to be covered by either one of the systems under equivalent traffic conditions which are explained in the following sections. The spectrum requirements in order to achieve the same delay probability are compared. Since any delay in accessing the network is undesirable in PMR applications, waiting time or reference delay is taken as zero. Hence the probability of delay, $\mathrm{P}(\mathrm{D}>0)$, is taken as the performance measure. The desirable delay probability is assumed to be less than 0.05 for both systems [5].

All mobile stations are assumed to use transmission trunking mode in which a user presses the push-to-talk button to initiate a call. With the TETRA system, when the call is established, an uplink channel in the initiator cell and downlink channels in cells where receiving group members exist, are allocated. The user releases the button after completing the transmission, and the channels are released.

In TETRA system, there is the provision that uplink and downlink channels can be allocated independently and not in uplink/downlink pairs. This capability corresponds to TETRA's best mode of operation in terms of efficiency in using the spectrum [13]. We assumed that this spectrally efficient allocation property is employed in TETRA, in our assessment.

The number of members in a group call is reported to be seven or more [18]. The total number of cells to which the members are distributed determines the required number of downlink channels in PMR systems [3]. Data on the number of cells engaged in a group call is not available. As the number of members in a group becomes larger or as cells in a service area become smaller in size due to heavy traffic load, the possibility of having the group distributed to a large number of cells increases. The total number of cells where downlink is required in a group call can be at most one less than the group size. We assumed that the number of cells involved in the downlink of a group call is 6 , one less than the minimum group size of 7 in our analysis. We have also discussed the consequences of having smaller number of cells in the call. With the a-PMR system, when the call is established, an uplink channel in the initiator cell and only a single channel in the broadband wide-area downlink are allocated. 
Spectrum requirement of any one of the systems is ultimately related to the traffic load in the system. In this section, first, basic definitions and assumptions regarding the relation between traffic load and spectrum requirement of the a-PMR system is formulated, and then the same is done for TETRA system. Finally the cellular structure under consideration is defined.

1) Spectrum Requirement of the a-PMR System: The following definitions are used for the a-PMR system with $N$ cells: $\boldsymbol{A}$ ind $_{j}^{\boldsymbol{u}} \quad$ The uplink traffic initiated in the jth cell due to individual calls.

$A_{-}$group $\boldsymbol{u}_{\boldsymbol{j}}^{\boldsymbol{u}}$ The uplink traffic initiated in the jth cell due to group calls.

$\boldsymbol{A}_{\_}$total $\boldsymbol{j}_{\boldsymbol{j}}^{\boldsymbol{u}}$ Total uplink traffic initiated in the jth cell.

$\boldsymbol{A}_{\text {_total }} \boldsymbol{d}^{\boldsymbol{d}}$ Total downlink traffic in the DAB downlink of the system. by

The total uplink traffic initiated in the jth cell can be expressed

$$
A_{-} \text {total }_{j}^{u}=A \_ \text {ind }_{j}^{u}+A_{-g r o u p}^{u}
$$

For any given individual or group call initiated in a cell, a single downlink channel must be allocated in the downlink of the a-PMR system. Therefore total downlink traffic is the sum of uplink traffic loads of all cells, i.e.

$$
A_{- \text {total }}{ }^{d}=\sum_{j=1}^{N} A_{-} \text {total }_{j}^{u}
$$

From $A_{-}$total ${ }^{d}$ one can find the total number of the required downlink channels using Erlang-C [19] formula for the desirable delay probability. Erlang-C formula is valid for Poisson distributed call arrivals and exponentially distributed call durations. Erlang-C formula further assumes that call arrivals are independent. When used in transmission trunking mode independence assumption for every transmission is not valid. However this model is still instrumental for gross capacity estimates in PMR systems [20]. Assuming $9.6 \mathrm{kbit} / \mathrm{s}$ bit rate for each channel, including coding and multiplexing overheads, one can then find the total spectrum needed for the downlink using T-DAB standards. From $A_{-}$total ${ }_{j}^{u}$, one can find the required number of uplink channels of the jth cell using Erlang-C formula for the desirable delay probability. Since a TETRA-like structure is assumed to be used in the uplink part of the proposed a-PMR system, $25 \mathrm{kHz}$ spectrum is assumed to accommodate 4 one way communications channels. The total uplink spectrum requirement of the system is the sum of spectrum requirements of all cells if there is no frequency re-use in the system. However if there is frequency re-use in the system, then the cluster structure must be taken into consideration.

In this study it is assumed that for an $N$ cell system all cells have the same individual and group uplink traffic loads, i.e. $A_{\text {_ind }} u=A$ ind $^{u}$ and $A_{\text {_group }}^{u}=A_{\text {_group }} u$. In this case,

$$
\begin{aligned}
& A_{\_} \text {total }_{j}^{u}=A \text { ind }^{u}+A_{\text {-group }} \\
& A_{\text {_total }}
\end{aligned}
$$

2) Spectrum Requirement of TETRA System: The following definitions are used for TETRA system with $N$ cells:

$\boldsymbol{A}$ ind $_{\boldsymbol{j}}^{\boldsymbol{u}} \quad$ The uplink traffic initiated in the jth cell due to individual calls.

$\boldsymbol{A}_{-}$group $_{j}^{\boldsymbol{u}}$ The uplink traffic initiated in the jth cell due to group calls.

$\boldsymbol{A}_{-}$total $\boldsymbol{j}_{\boldsymbol{j}}^{\boldsymbol{u}}$ Total uplink traffic initiated in the jth cell.

$A \operatorname{ind}_{j}^{d} \quad$ The downlink traffic in the jth cell due to individual calls.

$\boldsymbol{A}_{-}$group $_{j}^{\boldsymbol{d}}$ The downlink traffic in the jth cell due to group calls. $\boldsymbol{A}_{-}$total $_{j}^{d}$ Total downlink traffic in the jth cell.

Total uplink traffic in the jth cell is

$$
A_{-} \text {total }_{j}^{u}=A_{-} \text {ind }_{j}^{u}+A_{-} \text {group }_{j}^{u}
$$

and the total downlink traffic in the jth cell is

$$
A_{-} \text {total }_{j}^{d}=A_{-} \text {ind }_{j}^{d}+A_{\text {_group }}^{d} \text {. }
$$

The downlink traffic components of TETRA can be related to the uplink traffic components. Assuming individual uplink traffic in all cells are the same, i.e. $A_{-}$ind $_{j}^{u}=A$ ind $^{u}$ for $\mathrm{j}=1, \ldots, N$, one can show that $A$ ind $_{j}^{d}$ is also equal to $A-$ ind $^{u}$ as explained in Appendix A. Similarly, if uplink group call traffic in all cells are assumed to be the same, i.e. $A_{\text {_group }}^{u}=A_{\text {-group }}{ }^{u}$ for $\mathrm{j}=1, \ldots, N$, then, $A_{- \text {group }_{j}}^{d}$ can be related to $A_{-}$group ${ }^{u}$, as explained in Appendix B, as follows

$$
A_{\text {_group }}^{d}=M * A_{\text {_group }}{ }^{u}
$$

where $M$ is the average number cells involved in the downlink part of a group call. In summary, (5) and (6) are simplified to

$$
\begin{aligned}
& A_{-} \text {total }_{j}^{u}=A_{- \text {ind }^{u}}+A_{- \text {group }^{u}} \\
& A_{- \text {total }_{j}^{d}}=A \_ \text {_ind }^{u}+M \cdot\left(A \text { _group }^{u}\right)
\end{aligned}
$$

Both the uplink and downlink spectrum requirements of TETRA system are calculated similar to how the spectrum requirement of the uplink part of the a-PMR system is calculated.

3) Cluster Size: The total number of cells used in both a-PMR and TETRA systems is another important variable to be considered for making a comparison in terms of spectral efficiency, because larger number of cells allows for more efficient frequency reuse. In TETRA system, both uplink and downlink are based on a cellular structure. On the other hand, the a-PMR system has cellular uplink and single cell downlink. In order to find the number of cells, considering the carrier to interference ratio, appropriate cluster size should be calculated which is applicable to both systems. When hexagonal cells are assumed to be used and the service area is assumed to be flat, cluster size is determined from the desired carrier to interference ratio from the following formula [12];

$$
\frac{C}{I}=\frac{(3 N)^{\frac{\gamma}{2}}}{i_{o}}
$$

where $N, \gamma$, and $i_{o}$ stands for cluster size, path loss exponent and number of interferer cells respectively. The cells, which contribute to interference significantly in an un-sectored cellular system, are the six co-channel cells in the first tier [12], [21]. The effective number of interfering cells, however, depends on the traffic load in these cells and varies between $30 \%$ of this 
maximum in conventional PMR systems and $70 \%$ in heavily loaded trunked systems [3]. Furthermore the interference is only due to uplink from mobile stations in a-PMR and hence it must be lower compared to a standard cellular configuration. In this study, the effective number of interferer cells is assumed to be 3 in both systems, $50 \%$ of 6 , to avoid unfair comparison against TETRA and path loss exponent is taken as 4 . Since the minimum $\mathrm{C} / \mathrm{I}$ ratio is $19 \mathrm{~dB}$ in TETRA system, the appropriate cluster size is found to be 7 .

\section{B. Numerical Results}

In order to compare the a-PMR system with the standard TETRA system, one needs to define a common reference with respect to the traffic carried by both systems. In this study we first specified the amount of total capacity in the wideband downlink in the a-PMR system, as a reference. This means that we are specifying $A_{-}$total $^{d}$ for the a-PMR system. From $A_{-}$total $^{d}$ one can find $A_{-}$ind $^{u}$ and $A_{\text {-group }}{ }^{u}$ of a-PMR using (4), provided that we assume a certain relation between these variables. We have taken the percentage of group calls as $50 \%$ of the total as in the scenarios 8 and 9 in [5], which corresponds to security service requirements. We assumed that individual call traffic and group call traffic initiated in a cell are equal; $A$ ind $^{u}=A_{\text {-group }}{ }^{u}$.

It is natural to assume that the uplink traffic loads, $A$ ind ${ }^{u}$ and $A_{\text {-group }}{ }^{u}$, are the same for both a-PMR and TETRA. Therefore once $A_{-}$ind $^{u}$ and $A_{-}$group $^{u}$ are calculated for the a-PMR system as explained above, we use the same values for these variables for TETRA. From uplink traffic loads, $A$ _ind ${ }^{u}$ and $A_{\text {_group }}{ }^{u}$ values, one can then proceed to find the spectrum requirements of both the a-PMR and TETRA systems.

At first, the downlink capacity of the a-PMR system is taken as $2.3 \mathrm{Mbits} / \mathrm{s}$, which corresponds to the full channel bandwidth of $1.5 \mathrm{MHz}$ in T-DAB, and the spectrum requirements for both systems to handle the corresponding traffic are calculated. The calculations are then repeated for lower downlink capacities down to $100 \mathrm{kHz}$.

The 2.3 Mbits/s total downlink capacity of a $1.5 \mathrm{MHz}$ T-DAB channel corresponds to 240 traffic channels for downlink. This number is calculated by dividing the total capacity to the typical bit rate needed for a voice call. Using Erlang-C formula, with 240 channels and probability of delay of 0.05 , a-PMR system traffic capacity becomes 213.8 Erlang for the downlink part. The a-PMR system allocates one downlink channel for each uplink channel. Therefore the proposed system has equal uplink and downlink traffic of

$$
A_{-} \text {total }^{u}=A_{-} \text {total }^{d}=213.8 \text { Erlang }
$$

where $A_{-}$total ${ }^{u}$ stands for the total uplink traffic and $A_{-}$total $^{d}$ stands for the total downlink traffic in the network. Traffic generated per user in PMR systems is studied in [22] and also specified in ETSI scenarios [5]. For user profiles like that of public safety institutions in large cities, which usually have very large user populations, the traffic generated per user is given as 0.012 Erlang. On the other hand, it is reported that, mean call initiation rate is 3.79 per busy hour and mean message length is 15.7 seconds for a mobile station (vehicle) in a typical metropolitan police service in [22]. This statistics account for a larger two-way traffic of 0.0165 Erlang and we considered this level in our assessment. If we assume that half of this load is carried in either direction, 213.8 Erlangs of offered downlink traffic corresponds to a large population of 25915 users. Hence our selection of $100 \mathrm{kHz}$ to $1.5 \mathrm{MHz}$ downlink capacity is appropriate in our simulation studies.

The service area is assumed to be covered by 1 to 12 clusters each having 7 cells. Therefore spectrum efficiency comparisons are also repeated for different numbers of clusters.

1) Single-Cluster Case (7 Cells) Using 1.5 MHz Downlink Capacity $(M=6)$ : Using $1.5 \mathrm{MHz}$ capacity for the downlink part, for the 1-cluster case with 7 cells, traffic for each cell is calculated as

$$
A_{\text {_total }}^{u}=\frac{213.8}{7}=30.54 \text { Erlang }
$$

where $A_{-}$total ${ }_{j}^{u}$ stands for the total uplink traffic per cell

When a-PMR is used, $A_{-}$total $_{j}^{u}$ stands for the sum of $A_{-}$ind $^{u}$ and $A_{\text {_group }}{ }^{u}$ as in (3). Therefore the total uplink traffic per cell can be taken as 30.54 Erlang. Again using Erlang-C formula with this traffic load and $\mathrm{P}(\mathrm{D}>0)$ less than $0.05,41$ channels per cell are needed to handle uplink traffic. In the a-PMR system, a single carrier supports 4 channels; therefore 11 carriers per cell are required. This corresponds to a spectrum of $1.925 \mathrm{MHz}$ (11 carriers/cell $* 25 \mathrm{kHz} /$ carrier $* 7$ cells) for uplink purposes in addition to $1.5 \mathrm{MHz}$ allocated for the downlink part. Summing up these values, the total spectrum needed to handle $213.8 \mathrm{Er}$ lang by the a-PMR is found to be $3.425 \mathrm{MHz}$.

When TETRA system is used, total downlink traffic per cell can be calculated by (9). Since we have assumed that the individual and group call traffics are equal, (9) is simplified to

$$
A_{-} \text {total }_{j}^{d}=(M+1) \cdot A_{-} \text {group }^{u}
$$

where $A_{\text {-group }}{ }^{u}$ is equal to $30.54 / 2$ Erlang and $M$ is taken as 6 in our calculations.

The total downlink traffic per cell is found to be $106.9 \mathrm{Er}-$ lang from (11), which is significantly larger than 30.54, the uplink traffic of a-PMR cell. There is an increase in the traffic due the difference in the group call schemes. For 106.9 Erlangs and $\mathrm{P}(\mathrm{D}>0)$ less than $0.05,126$ channels per cell are needed to handle downlink traffic. This corresponds to 32 carriers per cell and a spectrum of $5.6 \mathrm{MHz}$ (32 carriers $/$ cell $* 25 \mathrm{kHz} /$ carrier $* 7$ cells) for downlink purposes. The spectrum needed for uplink is the same as in the a-PMR system, i.e. 1.925 MHz, since (3) and (9) are equal to each other. Therefore the total spectrum needed by the TETRA system to handle the same traffic carried by the a-PMR system is found to be $7.525 \mathrm{MHz}$. This bandwidth requirement is 2.2 times that of the a-PMR system.

2) Two-Cluster Case (14 Cells) Using $1.5 \mathrm{MHz}$ Downlink Capacity $(M=6)$ : For the two-cluster case with 14 cells, traffic for each cell can be calculated by

$$
A_{-} \text {total }_{j}^{u}=A_{-} \text {total }_{j}^{d}==\frac{213.8}{14}=15.27 \text { Erlang }
$$

When the a-PMR is used, the total uplink traffic per cell can be taken as 15.27 Erlang. Using Erlang-C formula with traffic 15.27 Erlang and $\mathrm{P}(\mathrm{D}>0)$ of $0.05,23$ channels, therefore 6 carriers per cell are required. This corresponds to a spectrum of $1.050 \mathrm{MHz}$ ( 6 carriers/cell $* 25 \mathrm{kHz} /$ carrier $* 7$ cells) for uplink purposes in addition to $1.5 \mathrm{MHz}$ allocated for the downlink part. 
TABLE I

SPECTRUM REQUIREMENTS FOR THE a-PMR SYSTEM WITH DIFFERENT CLUSTER CASES AND TRAFFIC

\begin{tabular}{|c|c|c|c|c|c|c|c|c|}
\hline $\begin{array}{c}\text { Capacity } \\
\text { allocated in } \\
\text { downlink }\end{array}$ & $\begin{array}{c}1 \text {-cluster } \\
\text { case }(\mathrm{MHz})\end{array}$ & $\begin{array}{c}2 \text {-cluster } \\
\text { case }(\mathrm{MHz})\end{array}$ & $\begin{array}{c}\text { 3-cluster } \\
\text { case }(\mathrm{MHz})\end{array}$ & $\begin{array}{c}\text { 4-cluster } \\
\text { case }(\mathrm{MHz})\end{array}$ & $\begin{array}{c}5 \text {-cluster } \\
\text { case }(\mathrm{MHz})\end{array}$ & $\begin{array}{c}\text { 6-cluster } \\
\text { case }(\mathrm{MHz})\end{array}$ & $\begin{array}{c}10 \text {-cluster } \\
\text { case }(\mathrm{MHz})\end{array}$ & $\begin{array}{l}\text { 12-cluster } \\
\text { case }(\mathrm{MHz})\end{array}$ \\
\hline $100 \mathrm{kHz}$ & 0.450 & 0.275 & 0.275 & 0.275 & 0.275 & 0.275 & 0.275 & 0.275 \\
\hline $200 \mathrm{kHz}$ & 0.550 & 0.550 & 0.375 & 0.375 & 0.375 & 0.375 & 0.375 & 0.375 \\
\hline $300 \mathrm{kHz}$ & 0.825 & 0.650 & 0.650 & 0.475 & 0.475 & 0.475 & 0.475 & 0.475 \\
\hline $400 \mathrm{kHz}$ & 1.100 & 0.750 & 0.750 & 0.750 & 0.750 & 0.575 & 0.575 & 0.575 \\
\hline $500 \mathrm{kHz}$ & 1.200 & 1.025 & 0.850 & 0.850 & 0.850 & 0.850 & 0.675 & 0.675 \\
\hline $600 \mathrm{kHz}$ & 1.475 & 1.125 & 0.950 & 0.950 & 0.950 & 0.950 & 0.775 & 0.775 \\
\hline $700 \mathrm{kHz}$ & 1.750 & 1.225 & 1.225 & 1.050 & 1.050 & 1.050 & 1.050 & 0.875 \\
\hline $800 \mathrm{kHz}$ & 1.850 & 1.500 & 1.325 & 1.325 & 1.150 & 1.150 & 1.150 & 0.975 \\
\hline $900 \mathrm{kHz}$ & 2.125 & 1.600 & 1.425 & 1.425 & 1.250 & 1.250 & 1.250 & 1.250 \\
\hline $1000 \mathrm{kHz}$ & 2.400 & 1.875 & 1.525 & 1.525 & 1.525 & 1.350 & 1.350 & 1.350 \\
\hline $1100 \mathrm{kHz}$ & 2.500 & 1.975 & 1.800 & 1.625 & 1.625 & 1.450 & 1.450 & 1.450 \\
\hline $1200 \mathrm{kHz}$ & 2.775 & 2.075 & 1.900 & 1.725 & 1.725 & 1.725 & 1.550 & 1.550 \\
\hline $1300 \mathrm{kHz}$ & 2.875 & 2.350 & 2.000 & 1.825 & 1.825 & 1.825 & 1.650 & 1.650 \\
\hline $1400 \mathrm{kHz}$ & 3.150 & 2.450 & 2.100 & 2.100 & 1.925 & 1.925 & 1.750 & 1.750 \\
\hline $1500 \mathrm{kHz}$ & 3.425 & 2.550 & 2.375 & 2.200 & 2.025 & 2.025 & 1.850 & 1.850 \\
\hline
\end{tabular}

Summing up those values, the total spectrum needed to handle 213.8 Erlang is found to be $2.55 \mathrm{MHz}$ by the a-PMR system.

When TETRA is used, total traffic per cell can be calculated

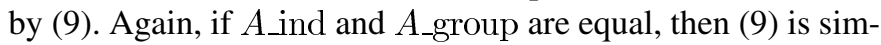
plified to (11) as in the single cluster case.

The total downlink traffic per cell is found to be $53.45 \mathrm{Er}-$ lang from (11). For this traffic and $\mathrm{P}(\mathrm{D}>0)$ less than 0.05 , 67 channels per cell are needed to handle uplink or downlink traffic. This corresponds to 17 carriers per cell and a spectrum of $2.975 \mathrm{MHz}$ ( 17 carriers/cell $* 25 \mathrm{KHz} /$ carrier $* 7$ cells) for downlink purposes. Therefore the total spectrum needed by the TETRA system to handle the same traffic carried by the a-PMR system is found to be $4.025 \mathrm{MHz}$.

3) Different Downlink Capacities and Number of Clusters $(M=6)$ : The bandwidth requirements of two systems are also compared for cases when smaller cell sizes are used for coverage. The service area is assumed to be covered by 3 clusters ( 21 cells), 4 clusters ( 28 cells), 5 clusters ( 35 cells) or up to 12 clusters ( 84 cells). As the number of clusters increase, the frequency re-use becomes more effective in TETRA. The spectrum requirement calculations are similar to those made for single and two-cluster cases.

Another comparison is made under lower traffic capacity requirement. Traffic that can be carried at downlink by a certain bandwidth of T-DAB is taken as reference, and the spectrum requirements of both systems to handle this traffic with $\mathrm{P}(\mathrm{D}>0)<0.05$ are determined.

Spectrum requirements of the a-PMR and TETRA systems under similar cellular coverage and traffic conditions are given in Tables I and II, respectively. The bandwidth allocated for downlink is given in the first column of both tables, which determines the traffic capacity of the a-PMR system. The total bandwidth requirements of either system to offer this traffic are calculated for larger number of clusters and are shown in the corresponding row of each table.

As expected, as the number of clusters increases, spectrum requirements of both systems decrease. This is mainly due to frequency reuse in the uplink part of the a-PMR system, and in both uplink and downlink parts of TETRA system. In order to compare the two systems for a given number of clusters and total traffic load, which also corresponds to total downlink capacity, we used the ratio of the spectrum requirement of the a-PMR system to the spectrum requirement of the TETRA system as the performance indicator. If the ratio is less than 1, then the a-PMR system is spectrally more efficient than the TETRA system, otherwise the reverse is true. Fig. 3(a) shows the spectrum requirement ratio for the single cluster case, versus the total capacity of the downlink of the a-PMR system. For the single cluster case, the average value for the spectrum requirement ratio is 0.45 , which means that the TETRA system requires 2.22 times the spectrum required by the a-PMR system. The dependence of spectrum ratio to allocated capacity has noisy behavior due to mainly two reasons, one of which is the quantization effect due to integer number of channels, and the other is the quantization effect due to the channel capacity of TETRA carriers, which is still a problem in the uplink part of the a-PMR system. A TETRA carrier employs multiples of 4 channels. Therefore, if 5 channels are needed in a cell, 8 channels will be allocated 
TABLE II

SPECTRUM REQUiREMENTS For TETRA SYSTEM With DifFERENT Cluster CASES AND TRAFFic $(M=6)$

\begin{tabular}{c|c|c|c|c|c|c|c|c}
\hline $\begin{array}{c}\text { Capacity } \\
\text { allocated in } \\
\text { downlink }\end{array}$ & $\begin{array}{c}1 \text {-cluster } \\
\text { case (MHz) }\end{array}$ & $\begin{array}{c}2 \text {-cluster } \\
\text { case (MHz) }\end{array}$ & $\begin{array}{c}3 \text {-cluster } \\
\text { case (MHz) }\end{array}$ & $\begin{array}{c}4 \text {-cluster } \\
\text { case (MHz) }\end{array}$ & $\begin{array}{c}5 \text {-cluster } \\
\text { case (MHz) }\end{array}$ & $\begin{array}{c}6 \text {-cluster } \\
\text { case (MHz) }\end{array}$ & $\begin{array}{c}10 \text {-cluster } \\
\text { case (MHz) }\end{array}$ & $\begin{array}{c}\text { 12-cluster } \\
\text { case (MHz) }\end{array}$ \\
\hline $100 \mathrm{kHz}$ & 0.875 & 0.525 & 0.525 & 0.350 & 0.350 & 0.350 & 0.350 & 0.350 \\
\hline $200 \mathrm{kHz}$ & 1.225 & 0.875 & 0.525 & 0.525 & 0.525 & 0.525 & 0.350 & 0.350 \\
\hline $300 \mathrm{kHz}$ & 1.750 & 1.050 & 0.875 & 0.700 & 0.525 & 0.525 & 0.525 & 0.525 \\
\hline $400 \mathrm{kHz}$ & 2.275 & 1.225 & 1.050 & 0.875 & 0.875 & 0.700 & 0.525 & 0.525 \\
\hline $500 \mathrm{kHz}$ & 2.625 & 1.750 & 1.225 & 1.050 & 0.875 & 0.875 & 0.525 & 0.525 \\
\hline $600 \mathrm{kHz}$ & 3.150 & 1.925 & 1.400 & 1.225 & 1.050 & 0.875 & 0.700 & 0.525 \\
\hline $700 \mathrm{kHz}$ & 3.675 & 2.100 & 1.575 & 1.225 & 1.050 & 1.050 & 0.875 & 0.700 \\
\hline $800 \mathrm{kHz}$ & 4.200 & 2.450 & 1.750 & 1.575 & 1.225 & 1.050 & 0.875 & 0.700 \\
\hline $900 \mathrm{kHz}$ & 4.725 & 2.625 & 1.925 & 1.575 & 1.225 & 1.225 & 0.875 & 0.875 \\
\hline $1000 \mathrm{kHz}$ & 5.250 & 2.975 & 2.100 & 1.750 & 1.575 & 1.225 & 1.050 & 0.875 \\
\hline $1100 \mathrm{kHz}$ & 5.600 & 3.150 & 2.275 & 1.750 & 1.575 & 1.225 & 1.050 & 0.875 \\
\hline $1200 \mathrm{kHz}$ & 6.125 & 3.325 & 2.450 & 1.925 & 1.750 & 1.575 & 1.050 & 1.050 \\
\hline $1300 \mathrm{kHz}$ & 6.475 & 3.675 & 2.625 & 2.100 & 1.750 & 1.575 & 1.050 & 1.050 \\
\hline $1400 \mathrm{kHz}$ & 7.000 & 3.850 & 2.625 & 2.275 & 1.925 & 1.750 & 1.225 & 1.050 \\
\hline $1500 \mathrm{kHz}$ & 7.525 & 4.025 & 2.975 & 2.450 & 1.925 & 1.750 & 1.225 & 1.050 \\
\hline
\end{tabular}

to that cell with redundant 3 channels. Fig. 3(b)-(f) display the dependence of spectrum requirement ratio to allocated capacity for 3, 4, 5, 6 and 10 clusters respectively. When the service area is covered with 4 or fewer clusters, the a-PMR system performs better for all allocated downlink capacity values. With 5 clusters, TETRA becomes more efficient as the allocated capacity is increased beyond $800 \mathrm{kHz}$. The reason is that both uplink and downlink parts of TETRA system are cellular, and therefore when higher number of clusters are used, the frequency reuse capability increases, thereby increasing the spectral efficiency. On the other hand, only the uplink part of the a-PMR system is cellular, therefore when higher number of clusters is used, the frequency reuse capability increases only for the uplink part. For 10 clusters, the spectrum requirement ratio is predominantly larger than 1 except for small capacity values. Fig. 4 shows the dependence of the spectrum requirement ratio to the number of clusters for 500,1000 and $1500 \mathrm{kHz}$ of downlink capacities. It is also clear from Fig. 4 that a-PMR is more efficient in networks which have up to 4 clusters, which corresponds to 28 cells.

4) Different Number of Cells (M) Engaged in Downlink: We studied the dependence of the spectral efficiency to the number of cells involved in the downlink of group calls, M, for different downlink bandwidth allocations. The results for $\mathrm{M}=$ $1, \ldots, 6$ and for downlink bandwidths of $500 \mathrm{kHz}, 1000 \mathrm{kHz}$ and $1500 \mathrm{kHz}$ are given in Table III(a), III(b) and III(c), respectively. For example, when the group members are assumed to be located in 3 different cells instead of 6 the a-PMR system is still spectrally more efficient for $1.5 \mathrm{MHz}$ downlink capacity and a network with single cluster. In this case, the ratio of the spectrum requirement of the a-PMR system to that of TETRA becomes 0.65 , instead of 0.45 . The ratio of bandwidth required in a-PMR to that required by TETRA decreases as $\mathrm{M}$ increases, whereas this ratio increases as the number of clusters increases. The a-PMR system remains efficient for up to 4, 3, 3, 2 and 1 clusters for $M=6,5,4,3$ and 2 , respectively when the downlink capacity is $1500 \mathrm{kHz}$. As M is decreased a-PMR is more advantageous over TETRA for smaller number of clusters. Similar observations are made for $500 \mathrm{kHz}$ and $1000 \mathrm{kHz}$ capacities. When $M=1$, a-PMR appears to be more efficient for a single cluster network, but this is due to the fact that TETRA has 4 channels per carrier, which causes a quantization effect. Otherwise two systems must perform similarly for a single cluster, and TETRA is more efficient for larger number of clusters. On the other hand, if the group members are distributed to a large number of cells a-PMR becomes more efficient for large number of clusters. For example, when $\mathrm{M}=10$ it is found that a-PMR is more efficient for up to 12 clusters for a $500 \mathrm{kHz}$ downlink.

\section{IMPLEMENTATION CONSIDERATIONS}

The implementation of a-PMR using systems with established standards is preferable. For example TETRA and T-DAB systems can be considered for this purpose. However both systems have different requirements regarding frequency bands, bandwidth, modulation schemes, channel coding, voice coding, compression, and frame structures. Also the medium access and control layer must be modified to accommodate an a-PMR system. This means that interfacing and internetworking are 


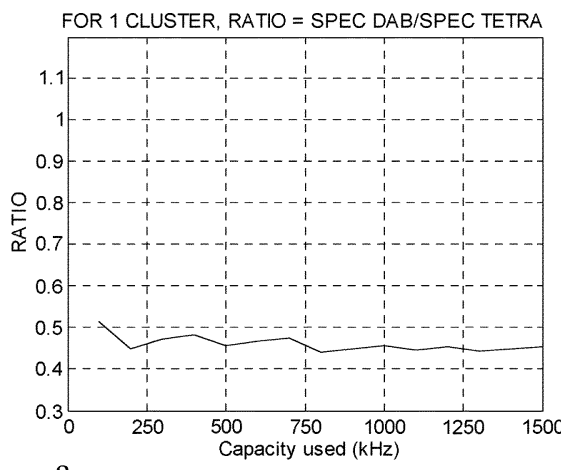

a
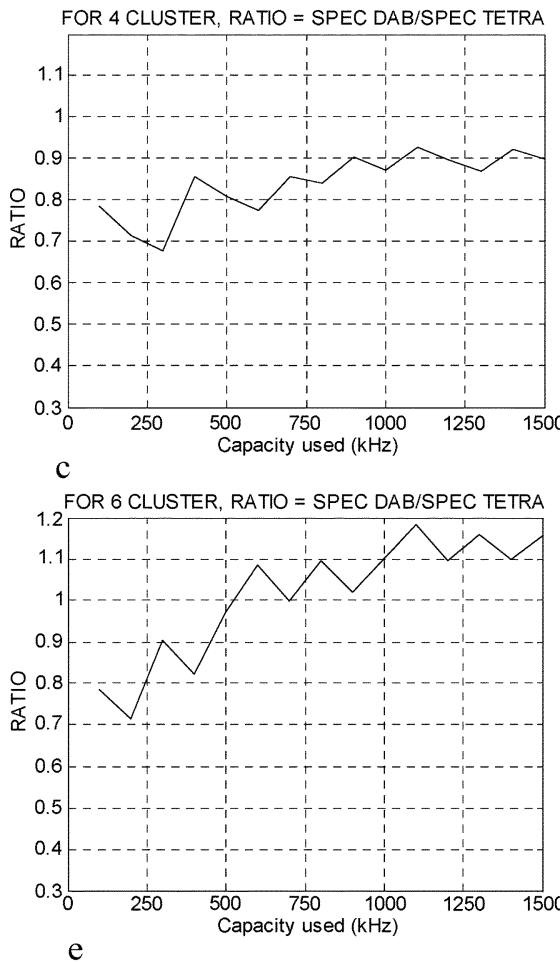

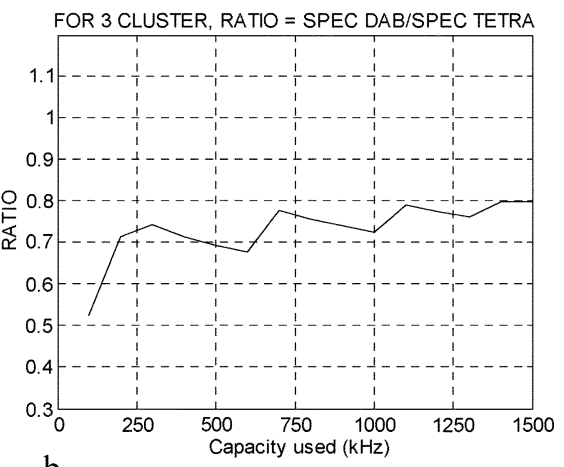

b

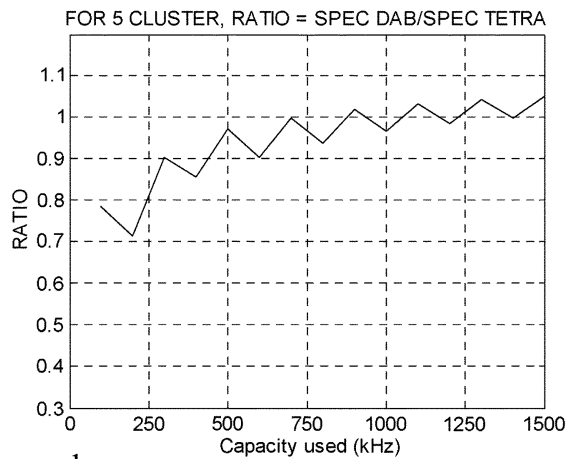

d
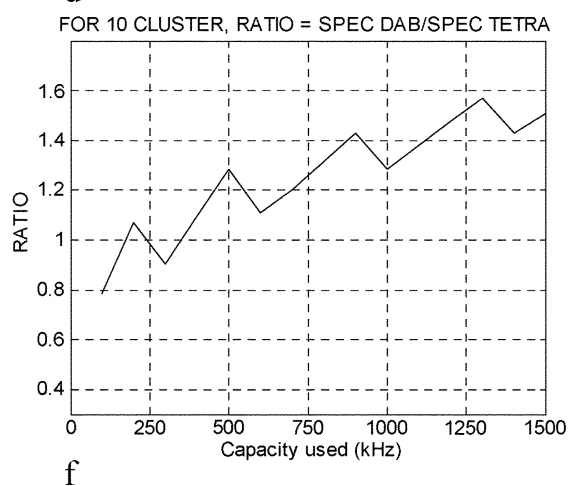

Fig. 3. Ratio of the total spectrum requirement of a-PMR to conventional TETRA system versus total traffic capacity of the downlink for (a) 1-cluster case, (b) 3-cluster case, (c) 4-cluster case, (d) 5-cluster case, (e) 6-cluster case and (f) 10-cluster case and $\mathrm{M}=6$.

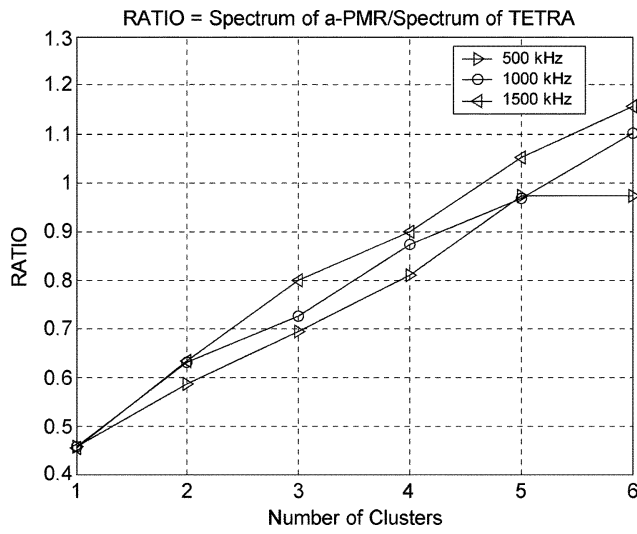

Fig. 4. Ratio of the total spectrum requirement of a-PMR to conventional TETRA system versus the number clusters in the system for different traffic loads and $\mathrm{M}=6$.

required. The emergence of standards of two systems preceded the vision and understanding of convergence of services in telecommunications, and therefore relevant facilities are not accommodated in existing standards. Applications like a-PMR, which require convergence of services, should be among the subject matters of the extensions of existing standards. There are several projects completed or still ongoing in the concept of the convergence of broadcast and cellular networks point of view. Therefore, as the interfacing and inter-networking between different systems become more realizable, the proposed system will be a promising solution to achieve spectrum efficient land mobile radio architectures.

Mobile tracking is necessary for the management of cellular uplink in a-PMR. In PMR systems this is maintained by mobile assisted hand-off. Since there is no base station downlink in a-PMR, mobile can not inform the system on the received power strength. In order to provide mobile tracking in a-PMR, a reversed procedure must be defined. When a mobile is switched $\mathrm{ON}$ it must acquire the information on all available uplink control channels. To provide this information to the mobiles relevant data must be continuously broadcast. Mobile then must transmit in these channels so that it can be assigned to the base station which receives the highest signal power. The system 
TABLE III

SPECTRAL EFFICIENCY COMPARISON FOR DifFERENT VALUES OF $M$ AND CLUSTER SizE. A) RATIO = SPECTRUM REQUIREMENT OF a-PMR/SPECTRUM REQUIREMENT OF TETRA FOR DOWNLINK CAPACITY OF $500 \mathrm{kHz}$. B) RATIO = SPECTRUM REQUIREMENT OF a-PMR/SPECTRUM REQUIREMENT OF TETRA FOR DOWNLINK CAPACITY OF $1000 \mathrm{kHz}$. C) RATIO = SPECTRUM REQUIREMENT OF a-PMR/SPECTRUM REQUIREMENT OF TETRA FOR DOWNLINK CAPACITY OF $1500 \mathrm{kHz}$

A)

\begin{tabular}{l|c|c|c|c|c|c|c|c}
\hline $\mathrm{M}$ & $\begin{array}{c}\text { 1-cluster } \\
\text { case }\end{array}$ & $\begin{array}{c}2 \text {-cluster } \\
\text { case }\end{array}$ & $\begin{array}{c}\text { 3-cluster } \\
\text { case }\end{array}$ & $\begin{array}{c}\text { 4-cluster } \\
\text { case }\end{array}$ & $\begin{array}{c}\text { 5-cluster } \\
\text { case }\end{array}$ & $\begin{array}{c}\text { 6-cluster } \\
\text { case }\end{array}$ & $\begin{array}{c}\text { 10-cluster } \\
\text { case }\end{array}$ & $\begin{array}{c}\text { 12-cluster } \\
\text { case }\end{array}$ \\
\hline 1 & 0.8571 & 0.9762 & 1.2143 & 1.2143 & 1.2143 & 1.2143 & 1.9286 & 1.9286 \\
\hline 2 & 0.6857 & 0.8367 & 0.9714 & 1.2143 & 1.2143 & 1.2143 & 1.2857 & 1.9286 \\
\hline 3 & 0.6234 & 0.8367 & 0.9714 & 0.9714 & 1.2143 & 1.2143 & 1.2857 & 1.2857 \\
\hline 4 & 0.5275 & 0.7321 & 0.8095 & 0.9714 & 0.9714 & 0.9714 & 1.2857 & 1.2857 \\
\hline 5 & 0.4898 & 0.6508 & 0.8095 & 0.8095 & 0.9714 & 0.9714 & 1.2857 & 1.2857 \\
\hline 6 & 0.4571 & 0.5857 & 0.6939 & 0.8095 & 0.9714 & 0.9714 & 1.2857 & 1.2857 \\
\hline
\end{tabular}

B)

\begin{tabular}{l|c|c|c|c|c|c|c|c}
\hline $\mathrm{M}$ & $\begin{array}{c}\text { 1-cluster } \\
\text { case }\end{array}$ & $\begin{array}{c}\text { 2-cluster } \\
\text { case }\end{array}$ & $\begin{array}{c}\text { 3-cluster } \\
\text { case }\end{array}$ & $\begin{array}{c}\text { 4-cluster } \\
\text { case }\end{array}$ & $\begin{array}{c}\text { 5-cluster } \\
\text { case }\end{array}$ & $\begin{array}{c}\text { 6-cluster } \\
\text { case }\end{array}$ & $\begin{array}{c}\text { 10-cluster } \\
\text { case }\end{array}$ & $\begin{array}{c}\text { 12-cluster } \\
\text { case }\end{array}$ \\
\hline 1 & 0.8571 & 1.0714 & 1.4524 & 1.4524 & 1.4524 & 1.9286 & 1.9286 & 1.9286 \\
\hline 2 & 0.7619 & 0.9740 & 1.0893 & 1.2449 & 1.4524 & 1.5429 & 1.9286 & 1.9286 \\
\hline 3 & 0.6531 & 0.8242 & 0.9683 & 1.0893 & 1.2449 & 1.5429 & 1.5429 & 1.9286 \\
\hline 4 & 0.5714 & 0.7653 & 0.8714 & 1.0893 & 1.0893 & 1.2857 & 1.5429 & 1.5429 \\
\hline 5 & 0.5079 & 0.7143 & 0.7922 & 0.9683 & 1.0893 & 1.1020 & 1.5429 & 1.5429 \\
\hline 6 & 0.4571 & 0.6303 & 0.7262 & 0.8714 & 0.9683 & 1.1020 & 1.2857 & 1.5429 \\
\hline
\end{tabular}

C)

\begin{tabular}{l|c|c|c|c|c|c|c|c}
\hline $\mathrm{M}$ & $\begin{array}{c}\text { 1-cluster } \\
\text { case }\end{array}$ & $\begin{array}{c}\text { 2-cluster } \\
\text { case }\end{array}$ & $\begin{array}{c}\text { 3-cluster } \\
\text { case }\end{array}$ & $\begin{array}{c}\text { 4-cluster } \\
\text { case }\end{array}$ & $\begin{array}{c}5 \text {-cluster } \\
\text { case }\end{array}$ & $\begin{array}{c}\text { 6-cluster } \\
\text { case }\end{array}$ & $\begin{array}{c}\text { 10-cluster } \\
\text { case }\end{array}$ & $\begin{array}{c}\text { 12-cluster } \\
\text { case }\end{array}$ \\
\hline 1 & 0.8896 & 1.2143 & 1.3571 & 1.5714 & 1.9286 & 1.9286 & 2.6429 & 2.6429 \\
\hline 2 & 0.7527 & 1.0408 & 1.2338 & 1.3968 & 1.6531 & 1.6531 & 2.1143 & 2.6429 \\
\hline 3 & 0.6524 & 0.8571 & 1.0440 & 1.2571 & 1.4464 & 1.4464 & 2.1143 & 2.1143 \\
\hline 4 & 0.5592 & 0.7669 & 0.9694 & 1.1429 & 1.2857 & 1.4464 & 1.7619 & 2.1143 \\
\hline 5 & 0.5018 & 0.6939 & 0.8482 & 1.0476 & 1.1571 & 1.2857 & 1.7619 & 1.7619 \\
\hline 6 & 0.4551 & 0.6335 & 0.7983 & 0.8980 & 1.0519 & 1.1571 & 1.5102 & 1.7619 \\
\hline
\end{tabular}

knows the position of the mobile at this stage, and the control channel data of neighboring cells can now be provided to the mobile for hand-off purposes. Mobile must regularly transmit at these control channels for hand-off purposes.

Direct mode of operation is also accommodated in PMR systems where two or more mobiles can communicate without using a base station. The a-PMR system does not address any issue on this mode and this facility must be available in handsets.

The transmission frames of T-DAB are organized into logical sub-channels to provide different audio broadcasting services. $\mathrm{T}$-DAB has provisions for reconfiguring the capacities allocated for different sub-channels. One sub-channel of T-DAB may be dedicated to carrying voice data of a-PMR, and the capacity spared for this sub-channel may be subjected to reconfiguration as the traffic load of a-PMR changes, for example, during the day. T-DAB standards, however, must be developed for assembling voice data into this dedicated sub-channel to take care of source identification, encryption and other requirements.

In T-DAB, consecutive samples of the source signal are spread over several transmission frames for the purpose of additional error correction. Due to this time-interleaving property, $384 \mathrm{~ms}$ delay occurs [16]. With this delay one can still be able to meet the "less than 1 second call set up time" requirement of a PMR system.

In conclusion, the above mentioned and other cross compatibility issues between the PMR and the broadcast system must be the subject matter of further detailed studies. Such studies are necessary for implementation and standardization, and are beyond the scope of this paper, which only considers possible gains in spectral efficiency.

\section{CONClusions AND Discussion}

A new asymmetric PMR system, which comprises a single broadband and wide area downlink cell and narrowband cellular uplink structure, is proposed in order to achieve higher spectral efficiency. When the broadband wide area downlink is implemented by means of a system similar to T-DAB, the downlink part becomes more robust and reliable. In a well planned OFDM based broadcast network the signal levels provided everywhere in the area can be significantly high and is well protected from multipath and interference. Furthermore, since the broadcast transmitters and their deployment procedures have good survival properties, they can be employed under emergency conditions.

We have shown that the proposed system is more efficient spectrally for less than or equal to 4 clusters if the average cells engaged in the downlink of a call is 6 . Due to relatively low traffic density in PMR applications, a PMR network with 4 clusters i.e. 28 cells can be assumed to adequately cover an average sized metropolitan area. In other words, if there should be 28 or fewer cells to cover a service area, the proposed system is shown to perform better than the TETRA system. Therefore the proposed system would be useful to achieve spectral efficiency 
in realistic system installations. As the number of clusters increases, the spectral advantage of the proposed a-PMR system is lost and this has been shown to be due to the fact that a symmetric standard PMR system can achieve more spectrum efficiency through frequency reuse.

We have shown that the presence of group calls is the main reason for achieving spectral efficiency through the proposed a-PMR system. The a-PMR system becomes more efficient as the number of cells involved in downlink increases. In our numerical studies we considered only group calls and individual calls. If the contributions of broadcast calls and open channel calls, which are received by all service users, are also considered, the spectral efficiency advantage of a-PMR increases further.

There are other factors that are to be considered for spectrum efficiency determinations. For example, in TETRA, almost $40 \%$ of both uplink and downlink capacity should be reserved for the traffic due to handoffs. However, in the proposed a-PMR system, spectrum reservations for handoff must be made only in the uplink part. Therefore, from the handoff point of view, the a-PMR system has additional advantage. Still another factor which influences the spectrum requirement of a PMR system is the need for additional control channel assignments for the purpose of call establishment and other control procedures. Again, in a-PMR there is need for control channels only for the uplink part. On the other hand, the downlink part of the a-PMR system will use some additional spectrum due to coding overheads which are needed for packet identification and multiplexing. Detailed inclusion of these additional factors could be the subject matter of further studies.

It is shown in this paper that the capacity provided by T-DAB within a $1.5 \mathrm{MHz}$ frequency band is sufficient to handle the downlink traffic requirement of a crowded PMR network. DVB-T is another means of providing wide area broadband downlink. In fact this system has a better potential for this kind of application, since it offers a gross data rate of up to $38 \mathrm{Mbit} / \mathrm{s}$ in $8 \mathrm{MHz}$ UHF TV channels [23]. Furthermore it has only 3 ms time interleaving depth which is significantly less than that of T-DAB. The analysis of using this capacity for spectrally efficient voice communication as well as potential problems in the implementation must be studied.

A broadband wide area downlink capability can also be considered as a supplement to a standard PMR installation. Emergency service requirements are fundamental in the development of PMR. It is argued that in emergencies, communication requirements are such that system must use dedicated channels and must not depend on more than one network. On the other hand, PMR systems are also used in many other applications. Availability of additional capability provided by a broadband wide area downlink can supplement the standard PMR installations in a spectrally efficient way particularly where user density is high.

If there are already T-DAB or DVB-T services installed in a metropolitan area, then some of the T-DAB or DVB-T spare capacity can be allocated to PMR services, and thus significant economic advantages can be obtained. Even if there are no already installed T-DAB or DVB-T services, the proposed system would still have economical advantages due to relatively low cost of T-DAB and DVB-T equipment. The proposed system has an additional advantage in implementing high data rate multimedia applications because of the availability of the broadband downlink. PMR systems are improving to provide high data rates in their evolution toward $3 \mathrm{G}$, such as in the case of TETRA Release 2 [24]. This development will also enable high data rates in the uplink part of the proposed convergence.

The a-PMR system obviously requires the development of new integrated handsets which combine all features of the PMR system and the receiver features of the broadcast system. Considering the low cost of digital broadcast integrated receiver electronics, which is a mature technology and which constitutes a large market, it is expected that there will be a small increase in the PMR handset cost. This issue, however, must also be carefully evaluated.

\section{APPENDIX}

\section{A. The Relation Between $A_{-} \operatorname{ind}_{j}^{d}$ and $A \_$ind ${ }^{u}$ in a Cell}

Assume there exist $N$ cells in the network and let $A$ ind $_{j}^{u}=$ $A$ ind ${ }^{u}$ denote the total individual uplink traffic in each cell in a TETRA network, where $\mathrm{j}$ denotes the jth cell and it is assumed that individual uplink traffic in all cells are the same and equal to $A$ ind ${ }^{u}$. Let $a$ be the probability that an individual call in a cell requests a downlink channel in the same cell and $(1-a)$ represent the probability that any other cell in the network is requested. Furthermore we assume that an individual call requests a downlink channel from other cells with equal probability $p=1 /(N-1)$. Then, total downlink traffic in a TETRA cell can be represented by

$$
\begin{aligned}
\text { A_ind }_{j}^{d} & =a \cdot A \_ \text {ind }_{j}^{u}+(1-a) \cdot \sum_{\substack{i=1 \\
i \neq j}}^{N} p \cdot A \text { ind }_{i}^{u} \\
& =a \cdot A \_ \text {ind } d^{u}+(1-a) \cdot(N-1) \cdot p \cdot A \_ \text {ind }^{u} \\
& =a \cdot A \_ \text {ind }^{u}+(1-a) \cdot(N-1) \cdot \frac{1}{(N-1)} \cdot A_{-} \text {ind }^{u} \\
& =A \_ \text {ind }^{u}
\end{aligned}
$$

\section{B. The Relation Between $A_{-} \operatorname{group}_{j}^{d}$ and $A_{-}$group $^{u}$ in a Cell}

Assume that there exist $N$ cells in the network and let $p_{j i}$ be the probability that the ith cell's group calls have a group member in the jth cell. All group members other than the transmitting one must receive the group call downlink. We assume that a group call downlink engages $M$ cells, where $M \leq N$, and that the initiation of group calls is uniformly distributed over the network. The probability $p_{j i}$ can be described by

$\begin{aligned} p_{j i}=P & \left\{\text { there exists a group member in the } \mathrm{j}^{\text {th }} \text { cell, }\right. \\ & \left.\text { given that a group call is initiated in the } \mathrm{i}^{\text {th }} \text { cell }\right\}\end{aligned}$

which is equal to

$$
\begin{aligned}
p_{j i}= & 1-P\left\{\begin{array}{c}
\text { cell } \mathrm{j} \text { is not engaged in cell } \mathrm{i}^{\prime} \text { s group call, } \\
\text { given that there is a group call in cell } \mathrm{i}\}
\end{array}\right. \\
= & 1-\frac{\left(\begin{array}{c}
N-1 \\
M
\end{array}\right)}{\left(\begin{array}{l}
N \\
M
\end{array}\right)}=\frac{M}{N}
\end{aligned}
$$


The relation between $A_{-}$group $_{j}^{d}$ and $A_{-}$group $_{j}^{u}$ in the jth cell can be represented by

$$
A_{\_} \operatorname{group}_{j}^{d}=\sum_{i=1}^{N} p_{j i} A \_ \text {group }_{i}^{u}
$$

which is also the expected value of the total downlink group call traffic of in the jth cell.

Since the uplink group call traffic are equal in all cells, i.e. $A_{\text {-group }}^{u}=A_{\text {-group }}$ for $^{\mathrm{i}}=1, \ldots, N$, the total downlink traffic in the jth cell can be described by

$$
\begin{aligned}
A_{\text {group }_{j}^{d}} & =A \_ \text {group }^{u} \sum_{i=1}^{N} p_{j i} \\
& =A \_ \text {group }^{u} \sum_{i=1}^{N} \frac{M}{N} \\
& =M \cdot\left(A \text { _group }^{u}\right)
\end{aligned}
$$

\section{REFERENCES}

[1] J. Dunlop, D. Girma, and J. Irvine, Digital Mobile Communications and the TETRA System: Wiley, 1999.

[2] Spectrum Efficient Digital Land-Mobile Systems for Dispatch Traffic (1998). [Online]. Available: http://www.itu.int/itudoc/gs/subscirc/itu-r/ 258-8.html. Report ITU-R M.2014.

[3] Methodology for the Assessment of PMR Systems in Terms of Spectrum Efficiency, Operation and Implementation (1997, Dec.). [Online]. Available: www.tetrapol.com/www/doc/Report_052_PMR_spectrum_efficiency.pdf. ERC Report 52.

[4] D. Kuypers, P. Sievering, and M. Steppler, "Traffic performance evaluation of TETRA and TETRAPOL," in Proc. 10th Aachen Symp. Signal Theory, Aachen, Germany, 2001, pp. 183-188.

[5] Scenarios for Comparison of Technical Proposals for MDTRS, ETSI (RES6.1), Jun. 1991

[6] R. Keller, T. Lohmar, T. Tonjes, and J. Thielecke, "Convergence of cellular and broadcast networks from a multi-radio perspective," IEEE Personal Commun., vol. 8, pp. 51-56, Apr. 2001.

[7] Digital Video Broadcasting (DVB): Interaction Channel for Digital Terrestrial Television Incorporating Multiple Access OFDM, EN 301 958, 2003.

[8] R. Rebhan, S. Olsson, B. Wergeland, P. Karlsson, and O. Franceschi, "Multimedia goes mobile in broadcast networks," IEEE Multimedia., vol. 4, pp. 14-22, Apr--Jun. 1997.

[9] T. Lauterbach and M. Unbehaun, "Multimedia Environment for Mobiles (MEMO)—interactive multimedia services to portable and mobile terminals," in ACTS Mobile Commun. Summit, vol. 2, Oct. 1997, pp. $581-586$.

[10] E. Sengul, B. Can, N. Akar, Y. Z. Ider, and H. Koymen, "Capacity analysis of a PMR system with DAB downlink," in 8th IEEE Symp.Computers and Communications (ISCC'2003), Antalya, Turkey, Jun. 2003.

[11] SABINA (System for Asymmetric Broadband Internet Access) project page. [Online]. Available: www.teracom.sequence

[12] T. S. Rappaport, Wireless Communications; Principles and Practice, 2nd ed: Prentice Hall, 2002.

[13] N. Boucher, The Trunked Radio and Enhanced PMR Radio Handbook: Wiley, 2000.

[14] J. H. Stott, "The how and why of COFDM," EBU Tech. Rev., 1998.

[15] R. V. Nee and R. Prasad, OFDM for Wireless Multimedia Communications: Artech House, 2000.

[16] Radio Broadcasting Systems; Digital Audio Broadcasting (DAB) to Mobile, Portable and Fixed Receivers, ETS 300 401, 2001.
[17] H. P. Stern, H. Sobol, and S. A. Mahmoud, "A narrowband, high capacity integrated voice/data mobile radio system for dispatch-related broadcas applications," IEEE Trans. Broadcast., vol. 41, no. 4, pp. 143-152, Dec. 1995.

[18] (1999, Dec.) Spectrum Demand for TETRA up to the Year 2008: Input to European Radiocommunications Office (ERO) DSI Phase III Consultation Process. Dolphin Telecommunications. [Online]. Available: www.ero.dk/eroweb/DSI-3/Dolphin.pdf

[19] L. Kleinrock, Queueing Systems, Volume 1: John Wiley \& Sons, 1975.

[20] G. C. Hess, Land-Mobile Radio System Engineering: Artech House, 1993.

[21] W. C. Lee, Mobile Cellular Communications, 2nd ed: McGraw-Hill, Inc., 1995.

[22] H. P. Stern, "Design issues relevant to developing an integrated voice/data mobile radio system," IEEE Trans. Veh. Technol., pp. 281-288, Nov. 1990.

[23] Digital Video Broadcasting Systems for Television, Sound and Data Services (DVB-T); Framing Structure, Channel Coding and Modulation for Digital Terrestrial Television, ETS 300 744, 2001.

[24] M. Edwards, "TETRA release 2.0 overview," in World Congress 2003 Seminar. led by the TETRA MoU Association, Available www.tetramou.com.

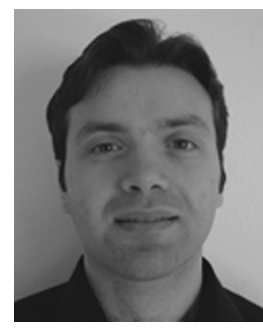

Ersin Sengül received the B.Sc. degree in electrical engineering from Middle East Technical University in 2001 and the M.Sc. degree in electrical engineering from Bilkent University in 2003. He is currently pursuing the $\mathrm{Ph} . \mathrm{D}$. degree in electrical engineering at the University of California, Irvine. His current research interests include design and performance analysis of multi-antenna systems for next generation wireless communications systems.

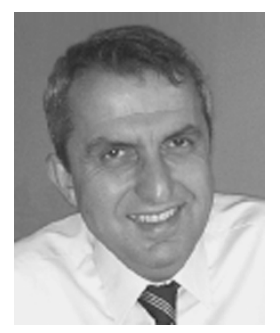

Hayrettin Köymen received the B.Sc. and M.Sc. degrees in electrical and electronic engineering from Middle East Technical University (METU) in 1973 and 1976, respectively, and the Ph.D. degree in 1979, from University of Birmingham, United Kingdom. He joined Institute of Marine Sciences, METU, Mersin, in 1979, and the EEE Department, METU, Ankara, in 1983. He has been with the EEE Department of Bilkent University since 1990. His current research interests are Underwater Acoustics, Mobile Communications and Spectrum Management.

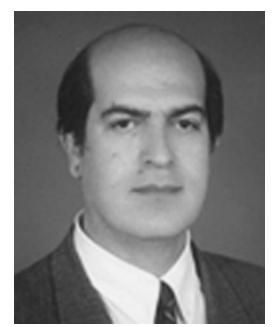

Yusuf Ziya İder received the B.Sc. degree in electrical engineering from Middle East Technical University in 1973, the M.Sc. degree in biomedical engineering from University of Southern California in 1976, and the Ph.D. degree in biomedical engineering from Northwestern University in 1979. He joined the EEE Department of Middle East Technical University in 1979, received Mustafa Parlar Foundation Young Investigator Award in 1986, and joined the EEE Department of Bilkent University in 2000 . His research interests are acquisition and processing of physiological signals, electrical impedance and magnetic resonance tomography, mobile communications and broadcast systems. 